\title{
Method for determining the calibration life of measuring devices. Case study - water meters
}

\author{
Mihaela Rodica Clinciu ${ }^{1}$, and Ramona Clinciu ${ }^{1, *}$ \\ ${ }^{1}$ Transilvania University of Braşov, Department of Manufacturing Engineering, Mihai Viteazu No. 5, \\ Braşov, Romania
}

\begin{abstract}
The paper presents the results of an experimental study conducted on specific measuring devices - water meters. The aims of the study are to determine the calibration life of the measuring devices as a function of the metrological reliability and to emphasize the parameter deviation as an important research tool for the quality assurance in metrology. The experimental study conducted is aimed at determining the calibration life of water meters of the same type and of precision class B. The research performed on the considered water meters can lead to the conclusion that the appropriate moment for performing the calibration for a specific set of measuring devices can be estimated by using the parameter deviation functions, these being obtained by means of regression analysis.
\end{abstract}

\section{Introduction}

Each measuring device is characterized by a certain tolerance interval. In case the measuring device is correctly adjusted, the measurement errors are located inside the tolerance interval. In time, by using the measuring device, the measurement errors might start to grow and, at a certain moment, they may exceed the tolerance interval. If the probability density function (pdf) for the measurement errors exceeds the tolerance interval with a certain percentage, it is considered that a metrological disturbance has appeared [1]. Metrological disturbances are difficult to discover at the moment they are produced, they can be discovered only during the periodical checking of the measuring device or by discovering the negative consequences generated by using the measuring device. In these conditions, it becomes very important to predict, with a certain precision, the moment when the measuring device measures with greater errors than the tolerated ones [1].

Parameter deviation represents an important research tool for the estimation of the calibration life of the measuring devices. It helps to predict the moment in time when the probability density function for the measurement errors exceeds the tolerance interval with a certain percentage, corresponding to the established level of significance. This moment corresponds to the stage in which the considered measuring device needs to be checked and, if necessary, calibrated $[1,2]$. This experimental research may be added to former researches performed on different types of measuring devices [3-5].

\footnotetext{
*Corresponding author: r.clinciu@unitbv.ro
} 
The present paper presents the results of an experimental study conducted on specific measuring devices - water meters of precision class B, a medium precision for the water meters. The experimental data is obtained in laboratory conditions and it is represented by the measuring errors, obtained by measuring the same volume of water by a single person, experimental data being grouped in samples produced at a certain interval of time.

The calibration life of the water meters, based on the parameter deviation functions, was estimated by using an appropriate algorithm. The estimated calibration life, obtained as a function of the metrological reliability, is compared to the requirements set in the appropriate standards. In the case of the water meters analysed, the estimated calibration life proved to be less than the one set in the standards, this fact meaning that the use of the respective water meters might lead to errors in metering.

\section{Estimating the calibration life of water meters}

General metrology uses especially the normal distribution, although positive and asymmetrical distributions, as Weibull distribution, are frequently met in the analysis of the metrological reliability [6].

The algorithm developed for estimation of the calibration life of the measuring devices uses the following notations: $\alpha$ - the level of significance; $m$ - the mean; $\sigma$ - the standard deviation; $n$ - the volume of the sample; $k$ - the number of samples; $x_{\mathrm{ji}}$ - the experimental data, which is assumed to be normally distributed, according to the normal distribution function $\mathrm{N}(m, \sigma, t)(j=1, \ldots, k, i=1, \ldots, n)$ having both parameters $m$ and $\sigma$ variable; $m_{0}$ the initial value of the mean; $a$ - the variation coefficient of the mean; $\sigma_{0}$ - the initial value of the standard deviation; $b$ - the variation coefficient of the standard deviation; $T_{\varepsilon}-$ the tolerance interval for variable $\varepsilon$, as a measurement error; $t i$ - the initial moment; $d t$ - the period of time at which samples are collected (it is considered that $d t$ is constant); $t_{\mathrm{r}}$ - the calibration time [2-4].

The case of a normal distribution $\mathrm{N}\left(m_{0}, \sigma_{0}, t\right)$ is considered. In time, due to the internal parameter deviation, the parameters of the distribution become $m$ and $\sigma$ and the normal distribution becomes $\mathrm{N}(m, \sigma, t)$. It is considered that the mean $m$ and the standard deviation $\sigma$ may have a linear or an exponential variation, according to the equations:

$$
\begin{aligned}
& m=m_{0}+a \cdot t \\
& \sigma=\sigma_{0}+b \cdot t \\
& m=m_{0} \cdot \exp (a \cdot t) \\
& \sigma=\sigma_{0} \cdot \exp (b \cdot t) .
\end{aligned}
$$

The algorithm for estimating the calibration life of the measuring devices as a function of metrological reliability includes the steps presented below [2]:

- Collecting experimental data (measurement errors);

- Performing data outlier tests- Grubbs test [7];

- Graphical representation (probability plot) and distribution assumption - normal distribution $[7,8]$;

- Parameter estimation for the normal distribution [7];

- Goodness-of-fit tests; it is considered a general goodness-of-fit test (KolmogorovSmirnov) and a normality goodness-of-fit test (Lilliefors). The optimum distribution is choosen by comparing the values obtained for the statistics of the tests considered with the critical values of the tests: $d_{\mathrm{j}}$ - the value of the statistics of the Kolmogorov-Smirnov goodness-of-fit test is to be compared to $d_{\mathrm{n}}, \alpha$ - the critical value of the statistics of the Kolmogorov-Smirnov goodness-of-fit test; $L_{\mathrm{j}}$ - the value of the statistics of the Lilliefors 
goodness-of-fit test is to be compared to $L_{\mathrm{n}, \alpha}$ - the critical value of the statistics of the Lilliefors goodness-of-fit test [6-8];

- Determining the parameter deviation functions by using regression analysis and the correlation coefficients for the linear variation and for the exponential variation $[7,8]$. The type of the parameter deviation function, linear or exponential, for parameters $m$ and $\sigma$, is indicated by the highest value of the two calculated correlation coefficients for each parameter;

- Determining the calibration life, using the parameter deviation functions and the method described in [2]. The period for the metrological control of water meters is set to seven years, as stated in standards [9].

\section{Experimental study}

The experimental study is conducted on water meters of the same type, of precision class B, a medium precision for the water meters, connected in series arrangement, at a water source (Figure 1). At the time the experimental study was conducted, all water meters were in their specified working period, they have been used in installations for a specific period of time, 34 months, and were still in their working period according to standards prescriptions [9].

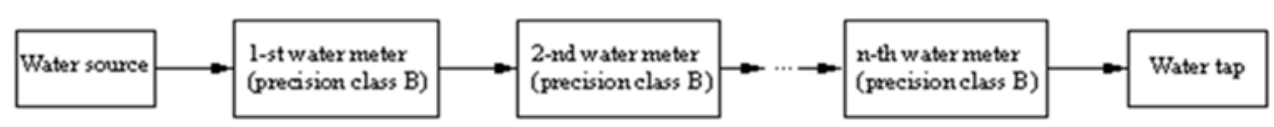

Fig. 1. Block scheme of the experimental installation.

The experimental data is represented by the measuring errors, obtained by measuring a certain volume of water, $0.01 \mathrm{~m}^{3}$. The measuring errors were obtained as the difference between the indications of each water meter used and the true value of the volume of water $\left(0.01 \mathrm{~m}^{3}\right)$. Data is grouped in eight samples produced at a certain period of time (a month), by measuring the same volume of water with the group of water meters, in laboratory conditions. Table 1 presents the measuring errors, obtained by measuring the same volume of water with the water meters considered. By analyzing the values of the errors, one can notice that they are all located within the appropriate tolerance interval.

Table 1. Measuring errors.

\begin{tabular}{|c|c|}
\hline Sample no. & Measuring errors $\left(\mathrm{m}^{3}\right)$ \\
\hline \multirow{2}{*}{1} & $-0.00007,-0.00011,-0.00006,-0.0002,-0.00011,-0.00004,-0.00016,0.00008$, \\
& $-0.00001,-0.00011,-0.00002,0.00002,-0.00007,-0.00012,-0.00003,-0.00001$ \\
\hline \multirow{2}{*}{2} & $\begin{array}{c}0.00011,0, .00011,-0.00006,-0.0002,-0.00011,0.0001,0,0.00005,-0.00008, \\
\end{array}$ \\
\hline \multirow{2}{*}{3} & $0.00017,-0.00008,0,0.00011,0.00007,0,0.00001$ \\
\hline \multirow{2}{*}{4} & $0.00001,0.00011,-0.000006,-0.0002,-0.00011,0.00004,0.00019,0.00008,-$ \\
& $-0.00011,0.00015,0.00016,0.00008,0.00003,0.00018,0.00007,0.00025,-$ \\
\hline \multirow{2}{*}{5} & $0.00004,0.00004,-0.00003,0.00002,-0.00002,0.00005,-0.00011,-0.00015$ \\
\hline \multirow{2}{*}{6} & $-0.00011,0.00015,0.00016,0.00008,-0.00013,-0.00018,-0.00007,-0.00015$, \\
& $-0.00014,0.00014,0.00013,0.0002,0.0002,0.00015,0.00018,0.00019$ \\
\hline \multirow{2}{*}{7} & $0.00017,0.0002,0.00018,0.00019,0.0002,0.00019,-0.00019,0,0.0001$, \\
& $0.00012,-0.00011,-0.00005,-0.00009,0.0002,-0.00002,-0.00005$ \\
\hline \multirow{2}{*}{8} & $0.00019,0.0002,0.00017,0.0002,0.00018,0.00019,-0.00019,0,0.0001$, \\
& $0.00012,-0.00011,-0.00005,-0.00001,0.0002,-0.00009,-0.00002$ \\
\hline
\end{tabular}


The tolerated maximum measuring errors are $\pm 2 \%$ for cold water (if water temperature $<=30^{\circ} \mathrm{C}$ ) [9]. The tolerance interval is $T_{\varepsilon}=0.0004 \mathrm{~m}^{3}$, the level of significance is $\alpha=0.05$, the volume of the sample is $n=16$, the number of samples $k=8$, initial moment $t i=0$ and the interval between the moments at which samples are collected $d t=1$.

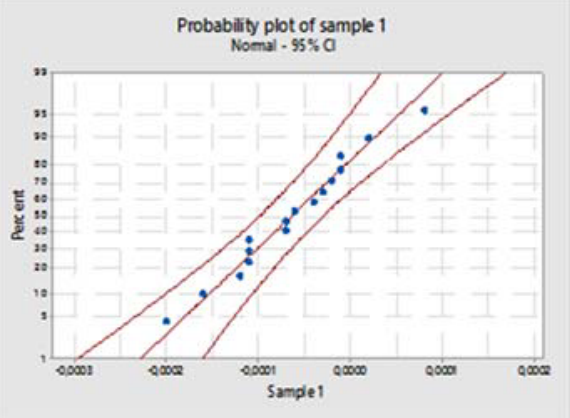

a

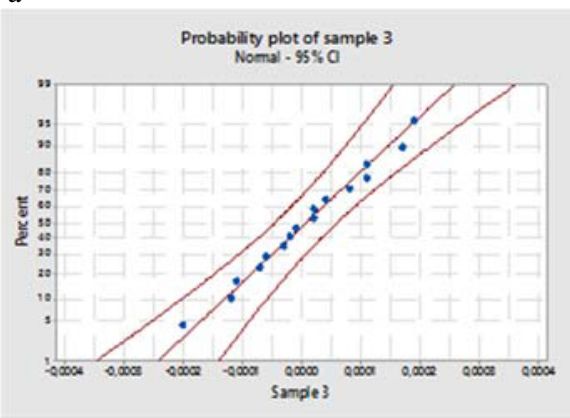

c

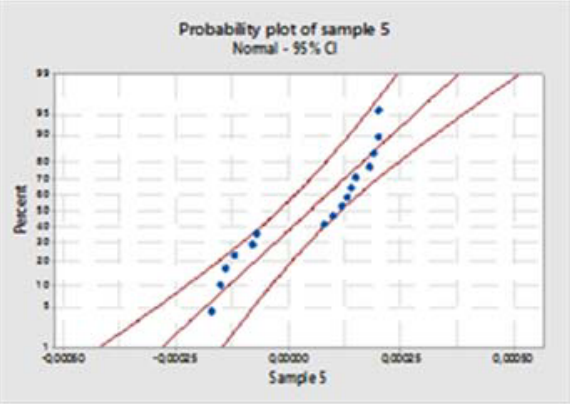

e

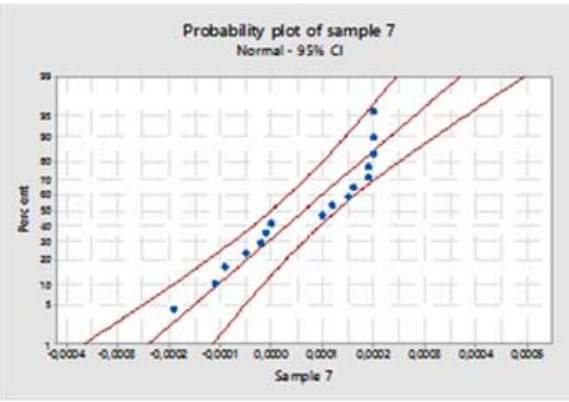

g

Fig. 2. Probability plots of the samples 1 to 8 .

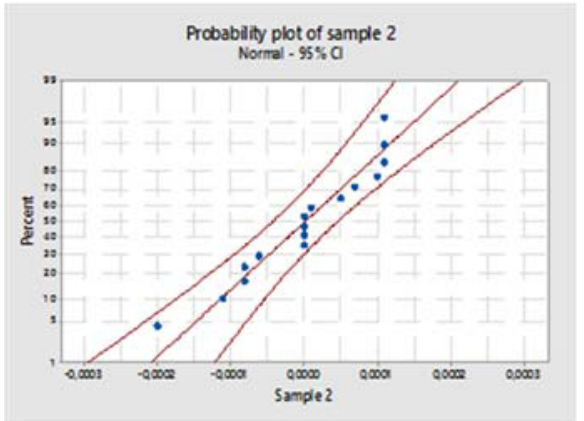

b

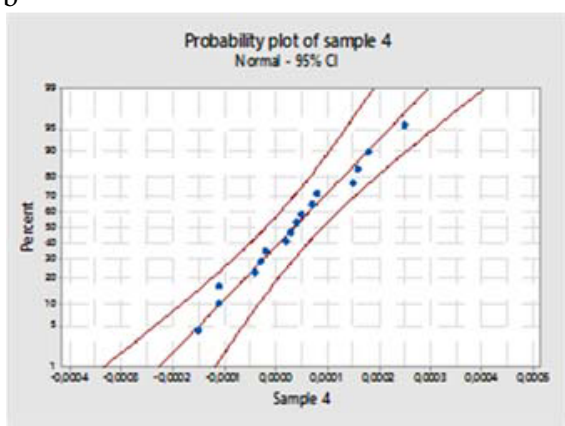

d

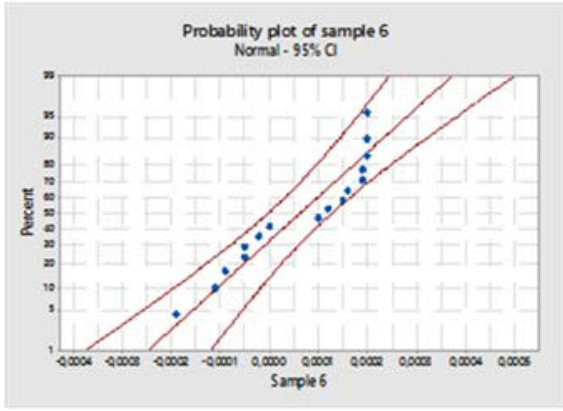

f

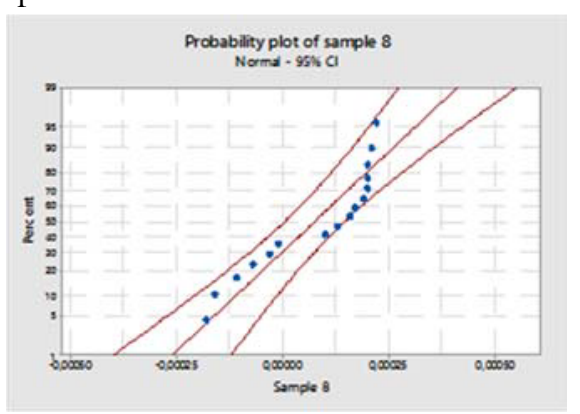

h 
The probability plots of the samples are presented in Figure 2, a to h. By analyzing the probability plots, one can assume that the distribution is the normal distribution and this fact is to be checked by using the goodness-of-fit tests.

The critical value of the statistic of the Kolmogorov-Smirnov goodness-of-fit test for the considered sample size is $d_{n, \alpha}=0.328$ and the critical value of the statistic of the Lilliefors goodness-of-fit test for the considered sample size is $L_{n, \alpha}=0.213$ [6-8].

Table 2. Values of the statistics of the goodness-of-fit tests.

\begin{tabular}{|l|c|c|c|c|c|c|c|c|}
\hline & \multicolumn{7}{|c|}{ Sample } \\
\cline { 2 - 9 } & 1 & 2 & 3 & 4 & 5 & 6 & 7 & 8 \\
\hline$d_{j}$ & 0.1191 & 0.1792 & 0.0797 & 0.0963 & 0.2676 & 0.2197 & 0.2175 & 0.2710 \\
\hline$L_{j}$ & 0.1191 & 0.1167 & 0.0787 & 0.0963 & 0.2051 & 0.1580 & 0.1591 & 0.2085 \\
\hline
\end{tabular}

Table 2 presents the values obtained for the statistics of both Kolmogorov-Smirnov and Lilliefors goodness-of-fit tests, for each sample. These values are according to both conditions: $d_{j} \leq d_{n}, \alpha=0.328$, for Kolmogorov-Smirnov goodness-of-fit test and respectively $L_{j} \leq L_{n}, \alpha=0.213$ for Lilliefors goodness-of-fit and this indicates normal distributions $[7,8]$.

Table 3. Values of the estimated parameters of the samples.

\begin{tabular}{|c|c|c|c|c|c|c|c|c|}
\hline & \multicolumn{7}{|c|}{ Sample } \\
\cline { 2 - 9 } & 1 & 2 & 3 & 4 & 5 & 6 & 7 & 8 \\
\hline$m$ & -0.00006 & 0 & 0.00001 & 0.00004 & 0.00005 & 0.00006 & 0.00007 & 0.00008 \\
\hline$\sigma$ & 0.00007 & 0.00009 & 0.00010 & 0.00011 & 0.00014 & 0.00013 & 0.00013 & 0.00014 \\
\hline
\end{tabular}

The values of the estimated parameters $m$ and $\sigma$ for each sample are given in Table 3 . By using the values of the means and standard deviations of the samples, presented in Table 3 , one can find the parameter deviation functions, by means of regression analysis [7, 8]. The correlation coefficients for linear variation of the parameters are $r_{\mathrm{m}-1}=0.9421$ and $r_{\sigma-1}=$ 0.9197 and the correlation coefficients for exponential variation of the parameters are $r_{\mathrm{m} \text {-exp }}$ $=0.9228$ and $r_{\sigma \text {-exp }}=0.9133$; these values indicate a linear variation for both parameters, according to the equations:

$$
\begin{gathered}
m=-0.00005+0.00002 \cdot t . \\
\sigma=0.00007+0.000001 \cdot t .
\end{gathered}
$$

The calibration life for the considered case is calculated by means of parameter deviation functions presented in equations (5) and (6), according to the method described in [2]. The value which is obtained for the calibration time for the case considered is $t_{\mathrm{r}}=$ 2.4029 time units (months), value which is less than the remaining period for the use of the water meters (water meters have been used for 34 months before the experiment), according to the pre-set period for the metrological control of the water meters, which is set to 7 years in the appropriate standards [9]. This fact means that the use of the respective water meters, more than the period indicated by the value $t_{\mathrm{r}}$, is possible to lead to errors in metering.

\section{Conclusions}

The method for determining the calibration life based on parameter deviation functions can be used for estimating the right moment for performing the metrological control/calibration for a specific set of measuring devices. This moment might be later than the pre-set one, and in this case financial costs with the metrological control are diminished, or earlier than 
the pre-set calibration time, and in this case greater errors than the tolerated ones are prevented.

By estimating the calibration life of the measuring devices as a function of metrological reliability, based on parameter deviation functions, it might be possible to test whether, for a certain type of measuring devices, the values obtained for the calibration life $t_{\mathrm{r}}$ are according to the requirements set in the appropriate standards. As future research, it is intended to consider other types of metrological devices, to test whether the values obtained for the calibration life meet the requirements set in the appropriate standards.

\section{References}

1. U. Wiener, F. Vereş, Quality of products and metrology J. 5, 265-271 (1972)

2. R. Clinciu, Bulletin of the Transilvania University of Braşov. 7(42), 65-68 (2000)

3. R. Clinciu, AMM. 371, 695-699 (2013)

4. R. Clinciu, AMM. 657, 694-698 (2014)

5. R. Clinciu, AJME. 12/1, 76-81 (2014)

6. V. Gh. Vodă, Applied metrology XXVI(1), 11-14 (1979)

7. I. Martinescu, I. Popescu, Reliability (Gryphon, Brasov, 1995)

8. D. Kececioglu, Reliability Engineering Handbook (Prentice Hall, Englewood Cliffs, N.J., 1991)

9. NML 003-05, Water meters (Monitorul Oficial al Romaniei, Bucuresti, 2005) 